\title{
Rehabilitación neuropsicológica en el síndrome de neglect tras accidente cerebro- vascular: caso clínico
}

Neuropsychological rehabilitation in neglect syndrome after stroke: case report

\author{
Modesto Romero-López ${ }^{1 *}$, Enrique Moraleda-Barreno ${ }^{1}$ \\ 1 Universidad de Huelva. Servicio de Neuropsicología y Rehabilitación. Huelva, España.
}

\section{Resumen}

El síndrome de neglect se manifiesta por déficits en la atención perceptiva y la respuesta a estímulos presentados en el hemicampo contralesional; frecuentemente es consecuencia de un accidente cerebrovascular que afecta al hemisferio cerebral derecho. Se presenta el caso de una paciente con infarto en la arteria cerebral media derecha a la que se le practicó una evaluación y participó en un programa de rehabilitación neuropsicológica. Se discuten la eficacia de la rehabilitación del neglect en sus diferentes aspectos.

Palabras clave: síndrome de neglect, anosognosia, accidente cerebrovascular, desinhibición conductual, rehabilitación neuropsicológica

\begin{abstract}
In this paper we approach two controversial issues to be found in the philosophical approaches to consciousness, the first one deals with the different uses of the terms "consciousness" and "to be aware of" so as to identify theirs uses which is unsettling for neurobiologists and philosophers. The difficulty lies in for neurobiologists in the concept of subjectivity, and for a philosopher in the category of qualia. The second one consists in upholding the thesis that puts into doubt the explicative character of the great majority of the materialist perspectives underlying current explanations of consciousness and qualia.
\end{abstract}

Keywords: neglect syndrome, anosognosia, stroke, behavioral desinhibition, neuropsychological rehabilitation

\footnotetext{
*Correspondencia: modesto.romero@dpsi.uhu.es Departamento de Psicología Clínica, Experimental y Social. Campus de "El Carmen", Universidad de Huelva. Avenida de las Fuerzas Armadas s/n, 21071, Huelva, España. Teléfono: +34 (959) 218329 . Fax. +34 (959) 210992

Recibido: 24-06-11. Revisión desde: 25-07-11. Aceptado: 25-11-11

DOI: $10.5839 / \mathrm{rcnp} .2011 .0602 .10$
} 


\section{Introducción}

En los accidentes cerebrovasculares (ACV) algunos pacientes muestran un síndrome característico que incluye negación de sus extremidades, acinesia, alteraciones en la atención, heminegligencia espacial, desviación de la cabeza y los ojos y negación explícita de su enfermedad denominado "síndrome de neglect" (Unsworth, 2007). Las tareas de evaluación y rehabilitación neuropsicológica pueden complicarse en aquellos pacientes que, además, presenten negación o falta de conciencia de déficits. El pronóstico viene dado por factores psicosociales, como el aislamiento social, edad avanzada, la gravedad de la hemiparesia, alteración de la memoria y la inteligencia, déficits visoperceptivos y reacciones emocionales inadecuadas.

En los pacientes que presentan síndrome de neglet se aprecia una rehabilitación más lenta e incompleta (Katz, HartmanMaeir, Ring \& Soroker, 1999). El tratamiento rehabilitador incluye las actividades de la vida diaria, condicionamiento espacial, entrenamiento en búsqueda visual junto a los aspectos motores afectados. Se han postulado diversos modelos explicativos de este síndrome, en los que la desinhibición y la conducta de utilización son frecuentes entre estos pacientes. Presentamos el caso de una paciente con síndrome de neglect, tras infarto cerebral isquémico, en el que se recogen todos estos aspectos implicados en el síndrome y su relevancia para la rehabilitación neuropsicológica.

\section{Caso clínico}

Mujer de 49 años que, tras intervención de cirugía cardíaca, presentó un infarto parieto-occipital derecho que requiere ventilación mecánica prolongada. Ya en planta hospitalaria la paciente estaba consciente, desorientada, con traqueostomía, hemiparesia derecha y paraparesias. Realizó rehabilitación motora con recuperación de fuerza en las extremidades, sin conseguir el control de esfínteres. La resonancia magnética cerebral mostraba un amplio infarto temporo-parieto-occipital derecho, con múltiples infartos lacunares en sustancia blanca fronto-parietal, localizada preferentemente a nivel periventricular y de ambas cápsulas internas.

Los resultados cuantitativos de la evaluación neuropsicológica a los cuatro meses del ACV se muestran en la tabla 1. La paciente colabora, se observa desinhibida, con conductas de utilización, apatía y falta de iniciativa. Sus actividades son muy reducidas y están referidas a las actividades básicas de la vida diaria, precisando ayuda y supervisión en todas ellas. No es capaz de usar dinero y se encuentra incómoda en todas las situaciones sociales.

Previo al ACV trabajaba como auxiliar de clínica y tenía entre sus aficiones las manualidades, costura y lectura. Los familiares refieren que, tras el ACV, de ser una persona pasional y de fuerte emotividad ha pasado a perder el interés por todo lo que no sean necesidades básicas, como comer y dormir, es menos cariñosa y no conserva sus amistades. Son escasos los episodios de conducta agresiva y destacan la falta de precaución en la deambulación por la ciudad.

De la evaluación neuropsicológica se concluye que, en la modalidad auditiva, no aparecen déficits en la función atencional simple, pero sí en la compleja, teniendo graves
Tabla 1. Resultados de las pruebas en la evaluación neuropsicológica.

\begin{tabular}{|c|c|}
\hline Pruebas & Resultados \\
\hline Mini-Mental Test & $\mathrm{PD}=22$ \\
\hline Test de trazado, modalidad A & 184 segundos - Errores $=4$ \\
\hline Test de trazado, modalidad B & Suspendido (incapaz de realizarlo) \\
\hline $\begin{array}{l}\text { Curva de memoria y aprendizaje (10 } \\
\text { ítems) }\end{array}$ & $\operatorname{IRR}=3.8-\operatorname{IRA}=2.9$ \\
\hline \multirow[t]{2}{*}{ Test de memoria Lógica } & Ejecución inmediata, $\mathrm{PE}=0$ \\
\hline & Ejecución diferida, $\mathrm{PE}=0$ \\
\hline Test de retención visual de Benton & Correctas $=1-$ Errores $=9$ \\
\hline Toni-2 & $\mathrm{PC}<4$ \\
\hline Test de Corsi & $P E=0$ \\
\hline Test de matrices de atención & $P E=0$ \\
\hline $\begin{array}{l}\text { Test de clasificación de tarjetas de Wis- } \\
\text { consin }\end{array}$ & $\begin{array}{l}\text { Suspendido (perseveración } \\
\text { continua) }\end{array}$ \\
\hline \multicolumn{2}{|c|}{ Escala de inteligencia de Wechsler (PT) } \\
\hline Información=10 & Figuras $=6$ \\
\hline Comprensión=2 & Claves $=3$ \\
\hline Aritmética $=8$ & Cubos $=0$ \\
\hline Semejanza=7 & Historietas $=5$ \\
\hline Dígitos $=8$ (directos $=5$; inversos $=3$ ) & Rompecabezas $=3$ \\
\hline Vocabulario $=7$ & $\mathrm{Cl}$ Manipulativo $=60$ \\
\hline Cl Verbal=79 & $\mathrm{Cl}$ Total $=67$ \\
\hline Escala de Barthel & $P D=80$ \\
\hline Dibujos & (Ver Figuras 1 y 2 ) \\
\hline
\end{tabular}

dificultades para realizar tracking mental. La atención visual y espacial está deteriorada en todas sus modalidades. Destaca la existencia de hemi-inatención espacial. Presenta graves problemas de desorientación espacial que afectan a su capacidad de deambulación. La desorientación temporal afecta con menor influencia en sus actividades diarias. La memoria visual y espacial está gravemente afectada. No obstante, conserva la capacidad de aprendizaje. En las capacidades visoconstructivas y visoperceptivas se observa apraxia en el vestir y apraxia constructiva. Estas alteraciones se ven agravadas por la hemiinatención visoespacial izquierda con predominio del cuadrante inferior (figura 1). Estos déficits la incapacitan para leer textos (lectura mecánica). Las funciones verbales son adecuadas para una conversación funcional.

La capacidad de planificación está muy deteriorada siendo incapaz de asimilar conceptos y realizar razonamientos, así como de solucionar problemas ejecutando las conductas necesarias. Su pensamiento se caracteriza por la impulsividad y la perseveraciones. La lesión ha provocado una disminución de la inteligencia, principalmente en la escala manipulativa, estando la verbal mejor conservada, siendo ambas patológicas.

La paciente muestra cierta consciencia de enfermedad. Los cambios en su personalidad y humor son referidos a episodios de aumento de irritabilidad, apatía, falta de responsabilidad, falta de iniciativa e interés, y pérdida de habilidades sociales. Destacan la desinhibición de conductas, la falta del control de impulsos y la conducta de utilización.

Desde la perspectiva de la rehabilitación neuropsicológica, se señala la complejidad del programa de intervención dada la variedad de funciones afectadas. La paciente participó en un programa de intervención multidisciplinar, centrado sobre todo lo relacionado con la integración de la información visual (ver figura 2). Dicho programa, diseñado al caso, consideraba las siguientes áreas en tareas individuales y de grupo:

- Motora: dirigida a potenciar las funciones sensitivas y motoras, mejorar la coordinación de movimientos, mejorar 
el equilibrio en bipedestación, aumentar la seguridad durante la marcha y mejorar la capacidad deambulatoria.

- Cognitiva: centrada específicamente en los aspectos cognitivos del neglect, habilidades atencionales, visoespaciales, de memoria, visuoconstructivas y de razonamiento. Los objetivos eran hacer consciente al paciente de su condición, usar estrategias compensadoras de búsqueda y generalizar esas estrategias a estímulos predecibles e impredecibles en la vida diaria. Los ejercicios consistían en tareas de papel y lápiz, sobre dibujos y mapas simples, ejercicios de navegación real (en interacción con el programa motor) y ejercicios de navegación virtual.

- Emocional: centrada en técnicas de modificación de conducta, extinción de la desinhibición y refuerzo de conductas socialmente apropiadas.

- De orientación a la familia: con el objeto de generalizar los aprendizajes al entorno familiar de la paciente.

\section{Discusión}

Algunos autores sostienen que los pacientes con negligencia pueden ser rehabilitados y que esta rehabilitación se puede transferir a diversas situaciones de la vida cotidiana (Lawson, 1962; Gianutsos, Cohen \& Batavia, 2004). Otros mantienen que la intervención terapéutica no es efectiva y no se generaliza; se pueden dar mejoras en las primeras semanas, pero posteriormente permanece estable (Gainotti, 1989, 1993). Otros, que no hay evidencia suficiente para afirmar o negar la efectividad de la rehabilitación (Luaute, Halligan, Rode, Rossetti \& Boisson, 2006). Otros apuntan al estudio definido por áreas en la rehabilitación para medir su efectividad: atención, percepción, función ejecutiva y funcionalidad en las actividades de la vida diaria (Zoccolotti, Cantagallo, De Luca, Guariglia, Serino \& Trojano, 2011).

Estas apreciaciones clínicas generales de la paciente y algunas específicas, como por ejemplo el salto a la página derecha antes de completar la izquierda, generalmente tras leer solo el primer reglón, parecen apoyar los modelos explicativos del sesgo atencional y falta de control inhibitorio ejercido por el hemisferio derecho en la dirección y selección de la atención (Posner, 1988, 1994). El síndrome de neglect se entendería como un problema de atención en la percepción. No obstante, no está claro el marco de coordenadas de referencia espacial afectado; respecto de la posición de la cabeza, al plano medio corporal o al centrado en el objeto (Hillis, Rapp, Benzing \& Caramazza, 1998; lachini, Ruggiero, Conso \& Trojano, 2009). Los estudios de procesamiento del tiempo podrían explicar, como hipótesis, la conducta de utilización y la impulsividad, por estar mediados por redes neurales de procesamientos distribuido, automático (Ivry \& Spencer, 2004) y sin control del tiempo prefrontal (Lewis \& Miall, 2006).

Este caso está en la línea de aquellos que sostienen la efectividad de la rehabilitación definida por objetivos (mejoras en la atención y percepción y en las actividades de la vida diaria), observándose mejoras incluso 12 meses después del ACV. No se ha obtenido mejoras en las funciones ejecutivas, posiblemente porque la red ventral no tiene la misma tasa de recuperación que la dorsal fronto-parietal (He, Snyder, Vincent, Epstein, Shulman \& Corbetta, 2007). Los últimos estudios realizados con adaptación de lentes para el control de la atención apuntan al papel del hemisferio derecho en el procesamiento explícito del tiempo, mientras que el izquierdo modula los efectos de estas adaptacio-

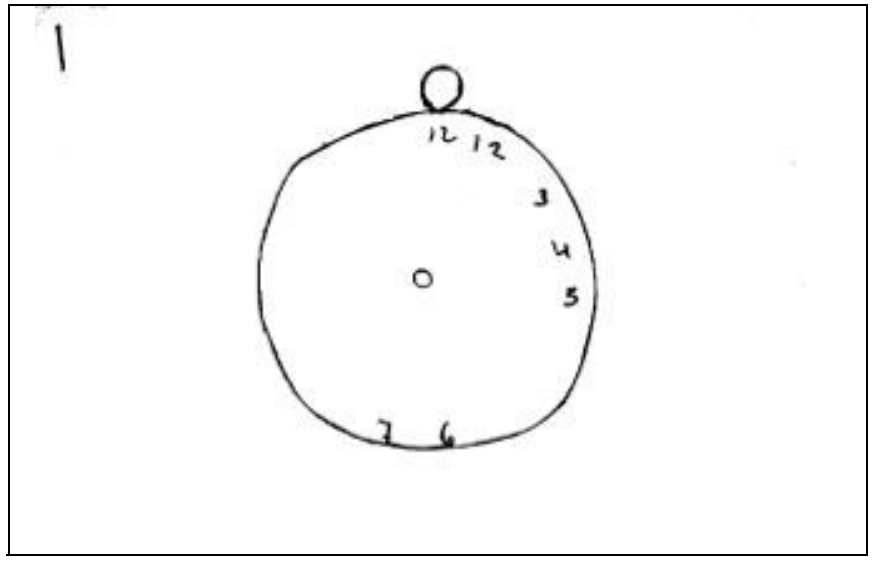

Figura 1. Test del dibujo del reloj realizado por la paciente.

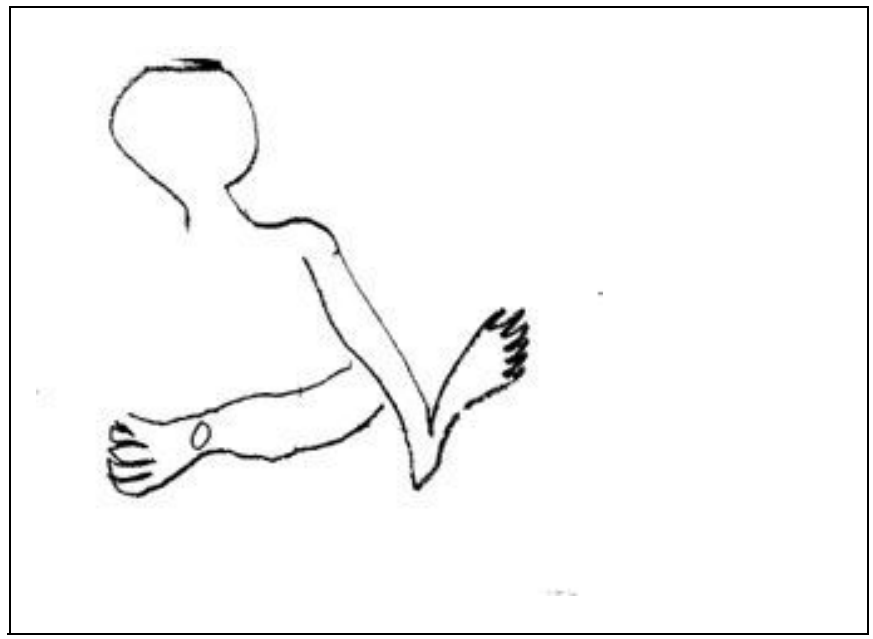

Figura 2. La paciente dibuja al neuropsicólogo durante la evaluación. Este se encuentra sentado, enfrentado y separado por la mesas de evaluación a la paciente. Le ha pedido que dibuje una figura humana.

nes en la percepción espacial y temporal (Magnania, Oliveri, Mancusod, Galantee \& Frassinetti, 2011). Con todo, se puede considerar el neglect como un síndrome complejo, en el que es posible la rehabilitación de diferentes aspectos del mismo, siendo necesarios programas específicos en los que la efectividad del tratamiento estará en función de los objetivos que se pretendan conseguir.

\section{Referencias}

Gainotti, G. (1993). Emotional and psychosocial problems after brain injury. Neuropsychological Rehabilitation, 3(3), 259-277.

Gaitnotti, G., Derme, P. \& Debonis, C. (1989). Clinical aspects and mechanisms of unilateral neglect. Revue Neurologique, 145(8-9), 626-634.

Gianutsos, J. G., Cohen, J.M. \& Batavia, M. (2004 ). Test-retest reliability in performance of persons with hemiparesis tracking by means of compatibly displayed myoelectric feedback derived from upper limb muscles. Perceptual and Motor Skills, 98(1), 19-30.

He, B. J., Snyder, A. Z., Vincent, J L., Epstein, A., Shulman, G. L. \& Corbetta, M. (2007). Breakdown of Functional Connectivity in Frontoparietal Networks Underlies Behavioral Deficits in Spatial Neglect. Neuron 53, 905-918.

Hillis, A. E., Rapp, B., Benzing, L. \& Caramazza, A. (1998). Dissociable Coordinate Frames of Unilateral SpatialNeglect: "Viewer-Centered" Neglect. Brain and Cognition, 37, 491-526. 
lachini, T., Ruggiero, G., Conson, M. \& Trojano, L. (2009). Lateralization of egocentric and allocentric spatial processing after parietal brain lesions. Brain and Cognition 69(3), 514-520.

Ivry, R. B. \& Spencer R. M. C. (2004). The neural representation of time. Current Opinion in Neurobiology, 14, 225-232.

Katz, N., Hartman-Maeir, A., Ring, H. \& Soroker, N. (1999). Functional disability and rehabilitation outcome in right hemisphere damaged patients with and without unilateral spatial neglect. Archives of Physical Medicine and Rehabilitation, 80(4), 379-384.

Lawson, I. R. (1962). Visual-spatial neglect in lesions of right cerebral hemisphere. Neurology, 12(1), 23-28.

Lewis, P. A. \& Miall, R. C. (2006). Remembering the time: a continuous clock. Trends in Cognitive Sciences, 10(9), 401-406.

Luaute, J., Halligan, P., Rode, G., Rossetti, Y. \& Boisson, D. (2006). Visuo-spatial neglect: A systematic review of current interventions and their effectiveness. Neuroscience and Biobehavioral Reviews, 30(7), 961-982.

Magnania, B., Oliveri, M., Mancusod, G., Galantee, E. \& Frassinetti, F. (2011). Time and spatial attention: Effects of prism adaptation on temporal deficits in brain damaged patients. Neuropsychologia, 49, 1016-1023.

Posner, M. I. (1994). Neglect and spatial attention. Neuropsychological Rehabilitation, 4(2), 183-187.

Posner, M. I., Petersen, S. E., Fox, P. T. \& Raichle, M. E. (1988). Localization of cog nitive operations in the human brain. Science, 240(1), 627-631.

Unsworth, C. A. (2007). Cognitive and Perceptual Dysfunction. En T.J. Schmitz \& S.B. O'Sullivan (eds.), Physical Rehabilitation (pp.1149-1185). Philadelphia, F.A: Davis Company.

Zoccolotti, P., Cantagallo, A., De Luca, M., Guariglia, C., Serino, A. \& Trojano, L. (2011). Selective and integrated rehabilitation programs for disturbances of visual/spatial attention and executive function after brain damage: a neuropsychological evidence-based review. European Journal of Physical and Rehabilitation Medicine, 47(1), 123-147. 